\title{
Traduire
}

Revue française de la traduction

229 | 2013

L'environnement, une spécialisation durable?

\section{Traduire dans le domaine des énergies renouvelables}

Éric Le Carre

\section{(2) OpenEdition}

Édition électronique

URL : http://journals.openedition.org/traduire/583

DOI : $10.4000 /$ traduire.583

ISSN : 2272-9992

Éditeur

Société française des traducteurs

Édition imprimée

Date de publication : 15 décembre 2013

Pagination : $36-40$

ISSN : 0395-773X

\section{Référence électronique}

Éric Le Carre, «Traduire dans le domaine des énergies renouvelables », Traduire [En ligne], 229 | 2013,

mis en ligne le 15 décembre 2015, consulté le 19 avril 2019. URL : http://journals.openedition.org/ traduire/583 ; DOI : 10.4000/traduire.583 


\section{Traduire dans le domaine des énergies renouvelables}

\section{Éric Le Carre}

La revue Traduire m'a aimablement invité à témoigner de mon parcours comme traducteur dans le domaine des énergies renouvelables. C'est précisément ce que je me propose de faire ci-après en retraçant les grandes étapes qui m'ont conduit à travailler régulièrement dans ce domaine.

\section{Les événements déclencheurs}

2007 : le débat sur la ratification du protocole de Londres et la détresse de nombreux collègues traducteurs de brevets confrontés à la disparition brutale d'une partie de leur activité, exprimée sur la liste d'échange interne de la SFT me font brutalement prendre conscience que rien n'est définitivement acquis et qu'il faut constamment se remettre en question et évoluer.

Cette détresse faisait aussi écho en moi car j'éprouvais alors de moins en moins de satisfaction à traduire des manuels informatiques et des interfaces de logiciels depuis quatorze ans déjà : j'avais l'impression d'en avoir fait le tour, mon intérêt pour ce type de traduction était moindre et les tarifs sur ce segment précis du marché commençaient leur inexorable mouvement de baisse.

En outre, j'avais envie de davantage traduire de l'allemand, ma deuxième langue de travail, par amour pour cette langue et l'Allemagne, pays avec lequel j'ai eu de nombreux contacts dans ma jeunesse. S'y ajoutaient des considérations économiques : le tarif est plus élevé que dans la combinaison de langues anglais - français, la concurrence est moindre et les pays germanophones ont une forte tradition de qualité.

J'étais également sûr de mes compétences en traduction technique et rédactionnelle en raison de mon grand nombre d'années d'expérience et des bons retours sur mon travail.

Oui, mais vers où aller et quel secteur choisir? 


\section{Le déclic}

En parcourant les étagères de ma bibliothèque, j'ai retrouvé l'ouvrage d'étude Wirtschaft Heute de mes premières années universitaires et suis alors tombé en arrêt devant le chapitre consacré à la protection de l'environnement en Allemagne. J'ai toujours été fasciné par la puissance du mouvement écologiste allemand et j'ai alors pris brusquement conscience du poids économique de ce secteur outre-Rhin. N'y avait-il pas là une piste à creuser?

Parallèlement, en France, nous étions au début de l'essor des énergies renouvelables. Les projets se multipliaient un peu partout sur le territoire. La presse locale se faisait l'écho de l'ouverture d'un parc éolien, de l'inauguration d'une nouvelle centrale solaire et en particulier dans ma région de la mise en service des toute premières installations de biogaz en milieu agricole. Je tenais là ma piste, mon nouveau domaine général de spécialisation et surtout un créneau potentiel.

\section{Les premiers pas}

À l'été 2007, j'ai profité d'un creux dans mon activité pour commencer à me documenter sur les énergies renouvelables et le biogaz en particulier. J'ai cherché sur internet des articles de fond en français et en allemand sur la technologie du biogaz, la filière en Allemagne, en France et en Bretagne, et ses acteurs.

J'ai alors décidé de créer un glossaire bilingue des termes de cette filière et un autre plus généraliste sur les énergies renouvelables plus classiques comme l'éolien et le solaire.

Je me suis aussi abonné à une revue professionnelle française et j'ai suivi l'actualité du secteur en France et en Allemagne au travers des flux RSS de sites spécialisés et de différentes lettres d'information.

\section{La démarche marketing}

J'ai élargi ensuite mes recherches pour voir s'il n'existait pas de petites agences de traduction spécialisées dans les énergies renouvelables, principalement dans les pays germanophones.

Après en avoir déniché quelques-unes, j'ai entrepris de les démarcher par e-mail en mettant en avant mon vif intérêt pour ce secteur et mes compétences et mon expérience en traduction technique et rédactionnelle.

Bien sûr, il m'a fallu montrer patte blanche et cela passait inévitablement par des tests de traduction non rémunérés qui se sont tous montrés concluants. Restait maintenant à attendre les premiers contrats, ce qui n'a pas tardé. 
Mais, certaines choses étaient claires pour moi dès le départ. Je ne voulais pas jouer les têtes brûlées au risque de remettre en cause ma crédibilité en tant que traducteur spécialiste du domaine et à terme ma collaboration avec ces agences.

Pour chaque mission, j'ai demandé à voir le texte au préalable pour déterminer son degré de difficulté. Inutile de prendre en charge des textes trop pointus. J'ai aussi privilégié les textes de clients pour lesquels il existait déjà des documents de référence, comme de précédentes traductions, une mémoire de traduction et/ou un glossaire. C'est un excellent moyen d'apprendre la terminologie du secteur. Côté tarifs, j'étais prêt à accepter certaines concessions. Mais finalement, je n'ai pas eu à les faire.

À partir de mes sources d'information spécialisées, j'ai commencé à mieux cerner les acteurs présents en France et en particulier dans ma région, mais aussi des entreprises étrangères de langue allemande récemment installées en France ou qui voulaient y exporter leurs produits.

Grâce à ces sources et à internet, j'ai identifié des interlocuteurs que j'ai démarchés par un message personnalisé de présentation de mes services.

Plus tard, j'ai visité des salons professionnels locaux et nationaux pour m'informer et essayer de trouver de nouveaux clients. Pour cela, je leur ai envoyé un message de présentation de mes services au préalable en annonçant ma visite sur leur stand.

\section{Les types de clients}

Les clients qui au final m'ont confié du travail directement ou par le biais d'agences de traduction spécialisées sont extrêmement diversifiés.

La plupart sont des fabricants de produits d'énergie renouvelable dans les domaines du solaire photovoltaïque et du biogaz.

J'ai aussi traduit pour des organismes nationaux et instituts d'études spécialisés dans les énergies renouvelables, ainsi que pour des associations professionnelles du secteur.

Dans une moindre mesure, j'ai travaillé pour un organisme spécialisé, commun aux gouvernements français et allemand, et pour l'édition française d'un magazine allemand spécialisé dans le solaire.

\section{Les types de textes}

À l'image des clients, les textes que j'ai traduits sont aussi extrêmement divers. Et même quand j'ai travaillé sur la durée pour un même client, ses textes se sont avérés très différents. 
Dans la majorité des cas, les textes avaient une dominante technique. Certains relevaient de l'ordre du rédactionnel avec une base technique. D'autres encore, mais bien plus rares, avaient une composante financière et/ou juridique.

Parmi les textes techniques figurent majoritairement des modes d'emploi d'installations ou de composants, des rapports techniques et, dans une moindre mesure, des rapports d'études économiques de certaines filières et des contrats. S'y ajoutent également des interfaces hommemachine de systèmes de commande.

Les textes rédactionnels sont constitués par les fiches produits, les caractéristiques techniques, les communiqués de presse et textes pour sites web, les articles de journaux et les présentations PowerPoint pour conférences.

\section{Bilan}

Le bilan personnel est évidemment positif puisque j'ai réussi à me diversifier dans ce segment du marché en traduisant régulièrement pour des entreprises du secteur des textes variés essentiellement en langue allemande.

Le bémol, c'est la difficulté de susciter l'intérêt d'un prospect étranger (le facteur de proximité est important) et la forte volatilité des clients directs. Très peu sont allés au-delà d'une mission de traduction ponctuelle. En outre, même avec des courriers personnalisés et ciblés, les retombées en termes de missions de traduction effectives ont été faibles, mais un nombre important des clients directs étrangers contactés a pris le temps de me répondre personnellement en indiquant leur intérêt pour mon profil.

Dans ce secteur, le traducteur est aussi tributaire de la politique en matière d'énergies renouvelables en vigueur dans son pays. La France est aujourd'hui moins attractive sur ce marché aussi, de sorte qu'en 2013, le flux des missions est moins important qu'il y a 4-5 ans.

e.lecarre@wanadoo.fr

Éric Le Carre est diplômé (en 1991) de l'École Supérieure d'Interprètes et de Traducteurs (ESIT) dans les combinaisons de langues anglais - français et allemand - français. Il a travaillé pendant onze ans comme traducteur spécialisé en localisation de logiciels et rédacteur technique chez différents éditeurs de logiciels internationaux et français.

Depuis 2004, il est traducteur indépendant spécialisé dans l'informatique, les énergies renouvelables, le marketing et la communication d'entreprise à Vannes (Morbihan). Membre actif de la section Grand Ouest de la Société Française des Traducteurs (SFT) depuis 2007, il anime également une téléformation sur la traduction des fichiers PDF pour le compte de SFT Services. En octobre 2013, il est devenu l'un des quatre délégués régionaux de la région Grand Ouest. 


\section{Témoignage}

Pendant mes études à I'ISIT, l'un de mes professeurs, l'inoubliable et avisée Mme Domercq, m'avait avertie : "la traduction dans le domaine du bois ? Non, vous subirez la concurrence des ingénieurs des Eaux et Forêts ! "...

Je suis issue du milieu sylvicole et j'ai longtemps pensé pouvoir percer dans ce secteur ainsi que celui du développement durable, domaine qui me plaît et dont je partage les valeurs.

Diplômée de l'ISIT en 2004, j'ai terminé mon cursus par un DESS de communication. Ce fut pour moi l'occasion de faire un stage à la Direction Développement Durable du groupe Carrefour. De fil en aiguille, j'ai fait de la traduction pour le Groupe en interne, puis en freelance. C'est ainsi que j'ai créé Traduction Durable. Me lançant à fond dans cette thématique, je m'imaginais traduire des rapports sur le développement durable ou des sites spécialisés. J'ai finalement traduit des chartes de DD, des évaluations de QSE(1), et suis devenue la traductrice (externe) d'une association luttant contre la désertification, entre autres.

En parallèle j'ai eu deux enfants et le souhait de leur consacrer du temps.

Mon démarchage en pâtit peut-être et ne fut pas si productif que prévu. Les agences me répondaient que ce n'était pas un domaine porteur, les associations ayant des budgets limités préféraient faire travailler leurs stagiaires et les institutions étaient difficiles à contacter.

Pourtant, l'idée ne devait pas être mauvaise puisque une agence m'a pris le nom que j'avais fait l'erreur de ne pas déposer !

Au bout de deux ans, estimant que la traduction n'était peut-être pas ma vocation, j'ai changé de métier pour être formatrice en entreprise. Je m'y sens très utile car, en personnalisant mes cours, je vois tout de suite les progrès des élèves.

Je suis heureuse de réaliser encore des traductions pour une grande agence qui me sollicite de temps en temps! La plupart des textes concernent les politiques de développement durable de groupes de luxe.

Ce que j'aurais dû faire pour que mon activité soit un succès : monter une agence avec une équipe de traducteurs de langues différentes, car le besoin à partir du français est indéniable, et être plus disponible pour mon travail.

Carole Vauthrin traductiondurable@gmail.com

(1) Qualité, sécurité, environnement. 